\title{
Virtuelle Mehrzeilen-CT-Bronchoskopie in der Diagnostik endobronchialer Tumoren: Fallbericht eines Karzinoidtumors
}

\author{
C. M. Heyer ${ }^{1}$ \\ T. T. Bauer ${ }^{2}$ \\ M. Orth ${ }^{2}$ \\ K.-M. Müller ${ }^{3}$ \\ V. Nicolas ${ }^{1}$ \\ G. Schultze-Werninghaus ${ }^{2}$
}

\author{
Virtual Multislice-CT-Bronchoscopy as a Diagnostic Tool in Patients \\ with Endobronchial Tumors: Case Report of a Carcinoid Tumor
}

\section{Zusammenfassung}

Hintergrund: Die Computertomographie (CT) spielt in der Bildgebung von Lungentumoren eine entscheidende Rolle. Mit der virtuellen Mehrzeilen-CT-Bronchoskopie ist es möglich, das Tracheobronchialsystem nicht-invasiv dreidimensional darzustellen. Bronchiale Karzinoide zählen zu den seltenen neuroendokrinen Lungentumoren. Wegen ihrer zentralen Lage erfolgt die Diagnose in der Regel bronchoskopisch-bioptisch. Kasuistik: Bei einem 70-jährigen Patienten mit Mischstaub-Pneumokoniose und chronischem Husten mit Hämoptysen zeigte sich - bei nativ-radiologisch überblähter linker Lunge - im Thorax-CT eine Raumforderung im anterobasalen Unterlappensegment. In der virtuellen dreidimensionalen CT-Bronchoskopie mittels SurfaceRendering wurde ein intraluminaler, okkludierender Tumor nachgewiesen, der konventionell endoskopisch und histopathologisch als Karzinoidtumor charakterisiert werden konnte. Virtuelle und konventionelle Bronchoskopie erbrachten deckungsgleiche Befunde. Therapeutisch erfolgte eine Lobektomie des linken Unterlappens mit mediastinaler Lymphadenektomie. Schlussfolgerungen: Die virtuelle Mehrzeilen-CT-Bronchoskopie kann bei endobronchialem Tumorwachstum wertvolle $\mathrm{Zu}$ satzinformationen über den Befund im Kontext seiner Umgebung liefern. Weitere prospektive Studien sind notwendig, um die Potentiale der virtuellen Mehrzeilen-CT-Bronchoskopie im Hinblick auf intraluminales Wachstum von Lungentumoren valide abschätzen zu können.

\section{Abstract}

Background: Bronchial carcinoids are rare neuroendocrine tumors of the lung. Diagnosis is established by computed tomography (CT) and bronchoscopy-guided biopsy. Imaging features of carcinoids are highly characteristic including contrast enhancement and central intraluminal growth. Multislice-CT generated three-dimensional virtual bronchoscopy enables non-invasive and detailed view of the tracheobronchial system. Case Report: A 70-year-old male with known silicosis and chronic cough with hemoptysis showed hypertransparency of the left lung on conventional images. Axial CT scans revealed a solid tumor in the left lower lobe. Virtual CT-bronchoscopy based on surface rendering depicted an intraluminal mass occluding the bronchus of the anterobasal segment that was confirmed by conventional endoscopy. Conventional and virtual bronchoscopy showed almost identical imaging features. Histopathological evaluation of the bioptic specimen revealed a typical carcinoid tumor. The patient successfully underwent resection of the left lower lobe with mediastinal lymphadenectomy. Conclusions: Virtual Multislice-CT-bronchoscopy can add important information about an intraluminal tumor and its relation to surrounding structures. Further studies have to be undertaken to evaluate the potentials of this promising method as a tool to determine endoluminal growth in tumorous lung lesions.

Institutsangaben

${ }^{1}$ Institut für Radiologie und Nuklearmedizin (Direktor: Prof. Dr. med. V. Nicolas), Berufsgenossenschaftliche Kliniken Bergmannsheil, Klinikum der Ruhr-Universität Bochum ${ }^{2}$ Abteilung für Pneumologie, Allergologie und Schlafmedizin (Leitender Arzt: Prof. Dr. med. G. Schultze-Werninghaus), Berufsgenossenschaftliche Kliniken Bergmannsheil,

Klinikum der Ruhr-Universität Bochum

${ }^{3}$ Institut für Pathologie (Direktor: Prof. Dr. med. K.-M. Müller), Berufsgenossenschaftliche Kliniken Bergmannsheil, Klinikum der Ruhr-Universität Bochum

Korrespondenzadresse

Dr. med. Ch. M. Heyer · Institut für Radiologie und Nuklearmedizin (Direktor: Prof. Dr. med.

Volkmar Nicolas) · Berufsgenossenschaftliche Kliniken Bergmannsheil · Klinikum der

Ruhr-Universität Bochum • Bürkle-de-la-Camp Platz 1 · 44789 Bochum

E-mail: christoph.m.heyer@ruhr-uni-bochum.de

Eingang: 12.12.02 - Nach Überarbeitung angenommen: 15.02 .03

Bibliografie

Pneumologie 2003; 57: 272-277 @ Georg Thieme Verlag Stuttgart · New York · ISSN 0934-8387 
Bildgebende Methoden haben einen festen Platz in der Diagnostik von Lungentumoren. Mit der virtuellen Mehrzeilen-CT-Bronchoskopie steht heute ein Instrument zur Verfügung, welches dreidimensionale, nicht-invasive Einblicke in das Tracheobronchialsystem erlaubt. Obwohl bislang prospektive Studien zur Validierung fehlen, zeichnet sich ab, dass die Methode bei intraluminal wachsenden Tumoren sinnvolle Zusatzinformationen liefern kann. Bronchiale Karzinoide zählen zu den seltenen neuroendokrinen Lungentumoren. Das histologische Spektrum reicht von niedrig-malignen, typischen Karzinoiden bis zu aggressiven, atypischen Tumoren. Da Karzinoide oft zentral liegen, erfolgt die Diagnose in der Regel bronchoskopisch-bioptisch. In Abhängigkeit von Größe und Histologie können die klinischen Symptome vielgestaltig sein.

Wir berichten exemplarisch über einen Patienten mit Hämoptysen und nativradiologisch einseitig heller Lunge, bei dem mittels virtueller CT-Bronchoskopie und Bestätigung durch konventionelle Endoskopie ein endobronchialer Karzinoidtumor histologisch gesichert werden konnte.

\section{Fallbericht}

Ein 70-jähriger Patient wurde wegen chronischen Hustens und Hämoptysen in unserer Klinik stationär aufgenommen. Nach 35-jähriger Unter-Tage-Tätigkeit im Bergbau war bereits eine Mischstaub-Pneumokoniose diagnostiziert worden. Bis auf eine Erhöhung des CA 19-9 auf 62 kIU/l (hausinterner oberer Grenzwert $<37 \mathrm{kIU} / \mathrm{l}$ ) waren die erhobenen Laborbefunde einschließlich Neuron-spezifischer Enolase (NSE), Cytoceratin Fraction 21-1 (CYFRA 21-1) und Squamous Cell Carcinoma Antigen (SCC-Ag) unauffällig.

Die p.a.-Thoraxaufnahme des Patienten zeigte - neben einzelnen klein-nodulären Formationen in beiden Lungen, vereinbar mit einer niedrig-gradigen Mischstaub-Pneumokoniose - eine erhöhte Strahlentransparenz der linken Lunge basal. Zudem demarkierte sich perihilär links eine ovale, weichteildichte Raumforderung (Abb.1). Das nach intravenöser Kontrastmittelgabe in Atemanhaltetechnik angefertigte Mehrzeilen-Spiral-CT (SOMATOM Volume Zoom ${ }^{\circledR}$, Siemens, Erlangen) zeigte auf den transversalen Schichten (Kollimation $4 \times 2,5 \mathrm{~mm}$, Schichtdicke $6 \mathrm{~mm}$ ) eine isolierte Überblähung des linken Lungenunterlappens bei

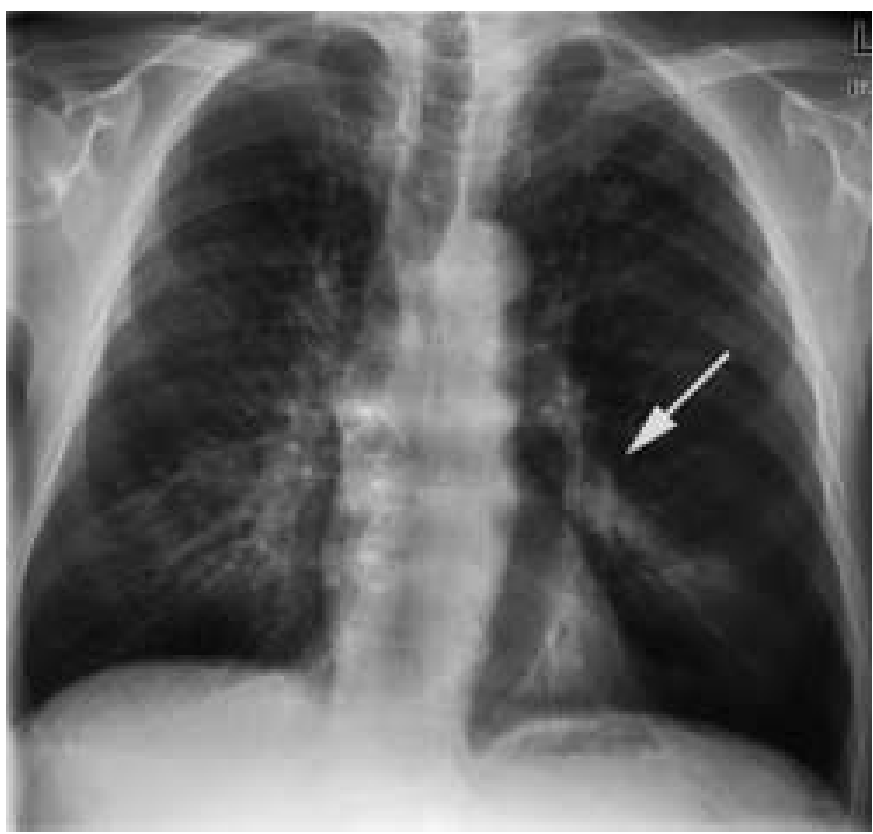

Abb. 1 Transparenzerhöhung des linken Lungenmittel- und -unterfeldes. Tumor links-subhilär (weißer Pfeil). Nebenbefund: MischstaubPneumokoniose

Nachweis eines zentral im anterobasalen Unterlappensegment gelegenen, nodulären und homogen perfundierten Tumor ohne Binnenverkalkungen (Abb. 2a u. b).

Aus dem in Spiraltechnik gewonnenen CT-Datensatz wurde eine virtuelle 3D-Bronchoskopie mittels Surface-Rendering errechnet (LEONARDO ${ }^{\circledR}$ Workstation, Siemens, Erlangen). Hier konnte eine sich in das Lumen des anterobasalen Unterlappensegment-Bronchus links (Segment 8) vorwölbende und diesen okkludierende Formation dargestellt werden (Abb.3a u.b). Der Befund wurde durch eine konventionelle Bronchoskopie bestätigt (Abb.3c). Eine Passage des Tumors nach distal gelang nicht.

Histopathologisch zeigten sich im Niveau des Schleimhautbindegewebes der Bronchialschleimhaut gelegene Tumorformationen mit positiver Reaktion für die neuroendokrinen Marker Synaptophysin, Chromogranin, Zytokeratin und CD56. Nach Markierung mit dem Proliferationsmarker MIB1 fanden sich nur geringe Proliferationen bei fehlenden Nekrosen und Mitosen. Es wurde die Diagnose eines typischen Karzinoidtumors gestellt. In der Somatostatin-Rezeptor-sensiblen Octreotid-Szintigraphie
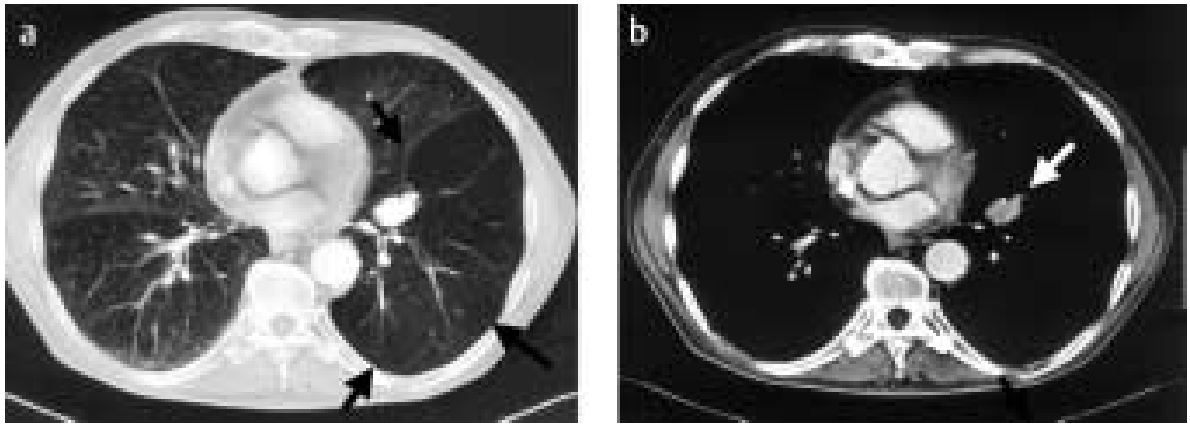

Abb. 2 Thorax-CT a) Lungenfenster: Isolierte Überblähung des linken Unterlappens (schwarze Pfeile), b) Mediastinalfenster: Tumorformation zentral im anterioren Unterlappensegment (weißer Pfeil) 

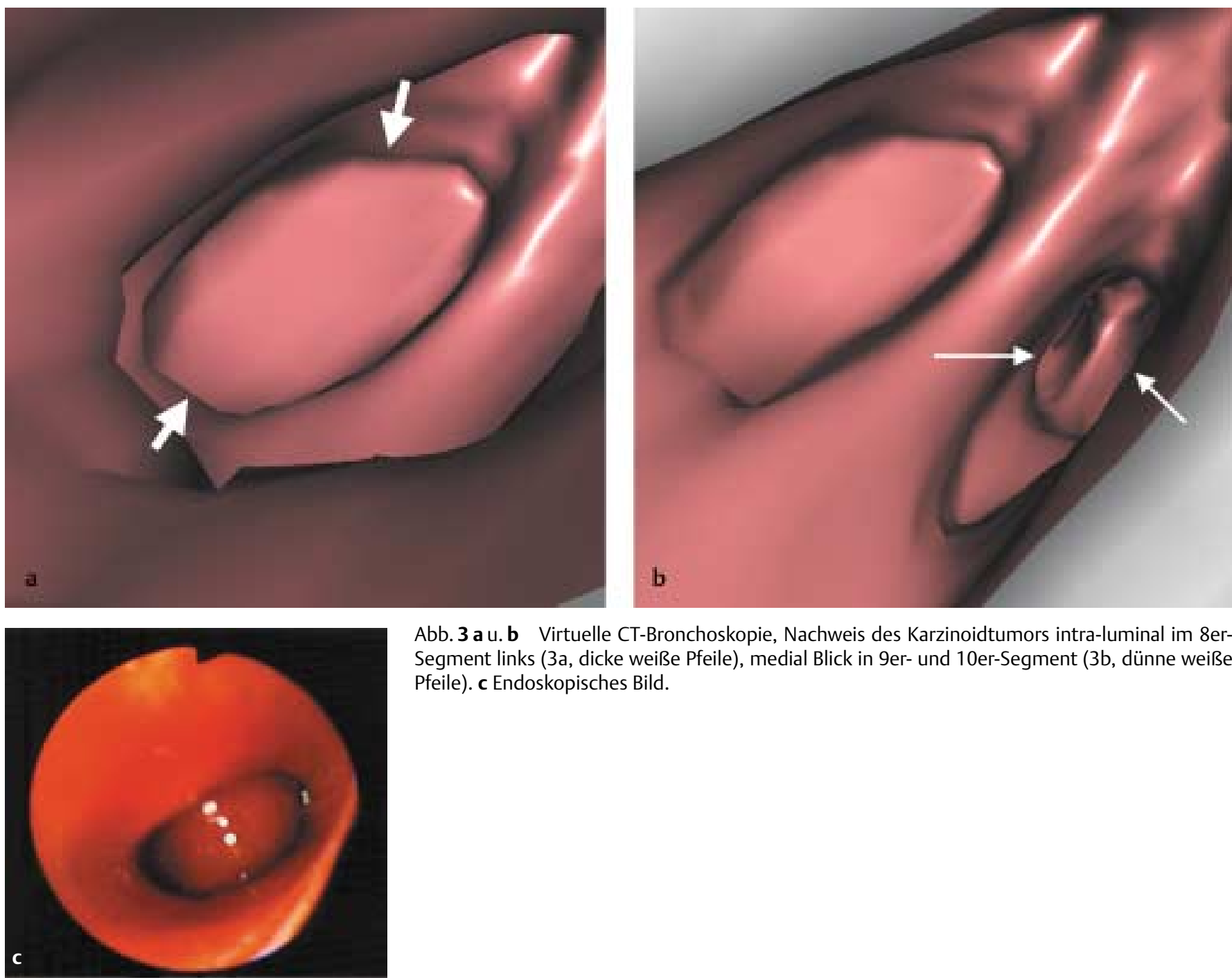

Abb. 3 a u. b Virtuelle CT-Bronchoskopie, Nachweis des Karzinoidtumors intra-luminal im 8erSegment links (3a, dicke weiße Pfeile), medial Blick in 9er- und 10er-Segment (3b, dünne weiße Pfeile). c Endoskopisches Bild.

mit Indium-111 demarkierte sich analog eine isolierte Mehranreicherung des intrathorakalen Tumors ohne weitere pathologische Nuklidfixationen.

Der Patient wurde einer operativen Therapie zugeführt, wobei eine Lobektomie des linken Unterlappens unter Mitnahme mediastinaler, links-hilärer und im Ligamentum pulmonale gelegener Lymphknoten erfolgte. Der postoperative Verlauf gestaltete sich komplikationslos.

Das gewonnene Präparat zeigte den rosaroten Tumor umgeben von schwarzem, anthrako-fibrotischem Lungengewebe. Die endgültige histopathologische Aufbereitung ergab einen proximal im 8er-Segment-Bronchus links gelegenen, bis $2 \mathrm{~cm}$ messenden typischen Karzinoidtumor mit Tumorinfiltrationen der Bronchialwand. Eine Gefäßinvasion konnte nicht nachgewiesen werden. In den tumorfreien Lymphknoten fand sich lediglich eine Anthrakose mit partieller Hyalinisierung (Abb.4).

\section{Diskussion}

Bronchiale Karzinoide zählen zu den malignen, neuroendokrinen Tumoren und machen 1-2\% aller Lungentumoren beim Erwachsenen aus [1]. Bei Kindern sind es die häufigsten primären Lungentumoren [2,3]. Das histologische Spektrum der Karzinoide ist breit und wurde 1999 von der WHO neu klassifiziert (Tab.1) [4]. Bei fehlender Geschlechtsbevorzugung liegt das durchschnittliche Alter betroffener Patienten bei 45 Jahren $[3,5]$. Aufgrund ihrer zentralen Lage rufen endobronchiale Karzinoide oft obstruktive Symptome wie Giemen, produktiver Husten, Fieber und asthmatische Beschwerden hervor [5,6]. Wegen des hohen Vaskularisierungsgrades der Karzinoide treten bei ca. 50\% der Patienten Hämoptysen auf [7]. 25-47\% der betroffenen Patienten sind bei Diagnosestellung asymptomatisch $[3,8]$.

Bildmorphologisch bieten karzinoide Tumoren oft einen typischen Befund: Die glatt begrenzten, ovalen Tumoren liegen in ca. $80 \%$ der Fälle zentral intra- oder peribronchial ohne segmentale Bevorzugung [5,9] bei einer Größe von 2 bis $5 \mathrm{~cm}$ [10]. Kleine Tumoren liegen oft vollständig intraluminal [11]. Multifokales Wachstum oder unscharf begrenzte Tumoren sind sehr selten $[8,9,11]$. Die für zentral gelegene Karzinoide typische bronchiale Obstruktion kann zur partiellen Verlegung des Bronchus und zur 


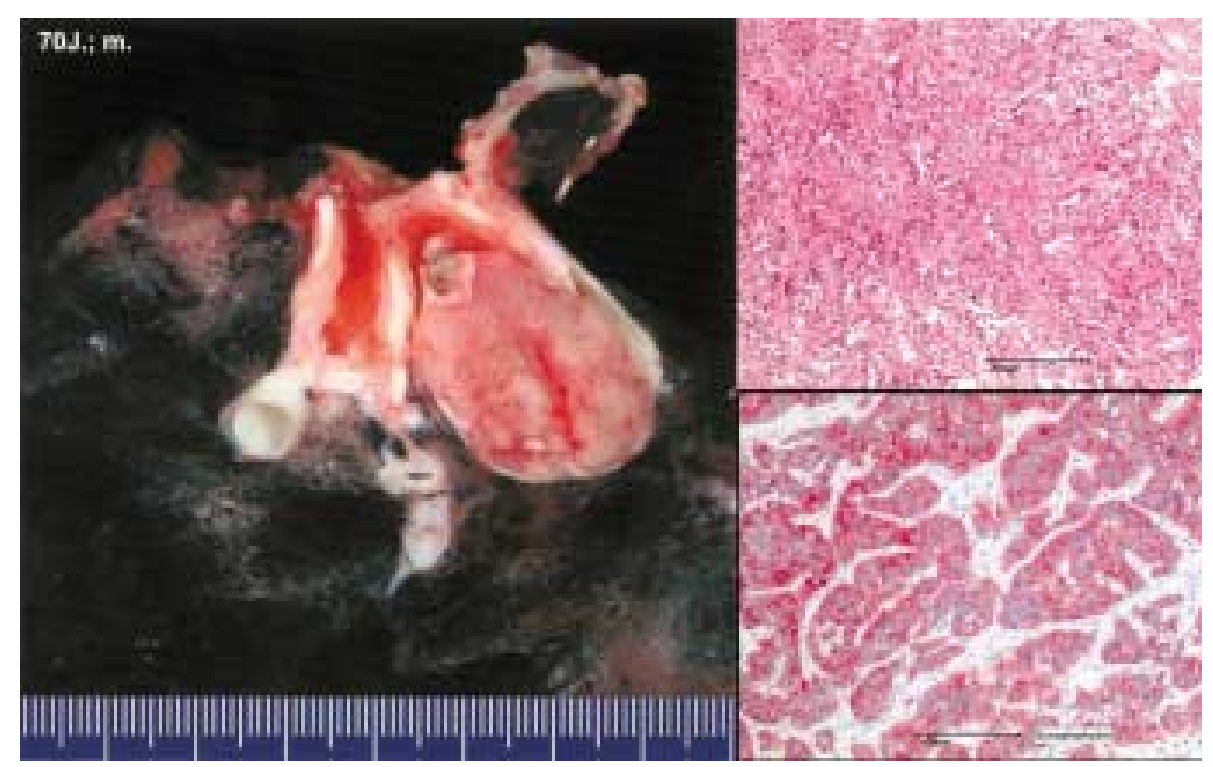

Abb. 4 Makroskopischer Befund des intraluminal gelegenen Tumors (links). HE-Färbung (rechts oben) und Immunhistochemie mit Synaptophysin (rechts unten)

Tab. 1 Synopse der WHO-Klassifikationen von 1981 und 1999 für Hyperplasien und Tumoren der Lungen mit neuroendokriner Differenzierung

\begin{tabular}{|c|c|c|c|}
\hline WHO 1981 & $I C D-O$ & WHO/IASLC 1999 & $I C D-O$ \\
\hline & & $\begin{array}{l}\text { diffuse idiopathic pulmonary } \\
\text { neuroendocrine cell hyperplasia } \\
\text { (DIPNECH) }\end{array}$ & \\
\hline \multirow[t]{5}{*}{ carcinoid tumour } & $8240 / 3$ & carcinoid tumour & $8240 / 3$ \\
\hline & & typical carcinoid & $8240 / 3$ \\
\hline & & atypical carcinoid & $8249 / 3$ \\
\hline & & $\begin{array}{l}\text { large cell neuroendocrine } \\
\text { carcinoma }\end{array}$ & $8013 / 3$ \\
\hline & & $\begin{array}{l}\text { combined large cell } \\
\text { neuroendocrine carcinoma }\end{array}$ & \\
\hline $\begin{array}{l}\text { small cell } \\
\text { carcinoma }\end{array}$ & $8041 / 3$ & small cell carcinoma & $8041 / 3$ \\
\hline oat-cell-carcinoma & $8042 / 3$ & & \\
\hline $\begin{array}{l}\text { intermediate } \\
\text { cell type }\end{array}$ & $8043 / 3$ & & \\
\hline $\begin{array}{l}\text { combined oat-cell } \\
\text { carcinoma }\end{array}$ & $8042 / 3$ & combined small-cell carcinoma & $8045 / 3$ \\
\hline
\end{tabular}

IASLC: International Association for the Study of Lung Cancer; ICD: International Classification of Disease; WHO: World Health Organization

Ventilstenose führen: Bei unbehinderter Inspiration wird eine suffiziente Exspiration durch Verlagerung des intraluminalen Prozesses verhindert; es kommt zur fokalen Lungenüberblähung. Die Ursachen einer einseitig „hellen Lunge“ im Röntgenbild sind vielfältig und beinhalten einseitiges oder lobäres Emphysem, überblähte Lungenzysten, angeborene Hypo- oder Aplasien einer Lungenarterie (z.B. bei Swyer-James-Syndrom), Lungenembolien, kompensatorische Lungenüberblähungen bei ipsioder kontralateraler Lungenlappenresektion, Thorax- oder Weichteilasymmetrien (z. B. nach Ablatio mammae) und intraluminale Fremdkörper/Tumoren. Da die Inzidenz intraluminaler Tumoren mit steigendem Lebensalter zunimmt, ist bei Erwachsenen mit einseitiger Lungenüberblähung daher immer eine Tu- morerkrankung auszuschließen. Lange bestehende Ventilstenosen können zu Schleimretention, rezidivierenden Infektionen und zur Ausbildung von Bronchiektasen führen [11].

In bis zu 30\% der Fälle weisen intrabronchiale Karzinoide Verkalkungen auf, die anhand der CT problemlos identifiziert werden können $[10,12]$. Kavernöse oder zystische Formationen treten selten auf [13]. Das Kontrastmittelverhalten karzinoider Tumoren ist unterschiedlich: Neben den häufigen gut vaskularisierten Formen kommen Tumoren vor, die kaum Kontrastmittel aufnehmen. Eine sichere Differenzierung von Karzinoiden und Bronchialkarzinomen ist aufgrund des Kontrastmittelverhaltens nicht möglich [14].

In ca. $20 \%$ der Fälle imponiert ein bronchiales Karzinoid als solitärer, peripher gelegener Lungenrundherd. Diese sind in der Regel glatt begrenzt oder lobuliert und oval bis rund; sie zeigen ein langsames Wachstum [9-11]. Karzinoide können mit vergrößerten mediastinalen Lymphknoten einhergehen, wobei diese in der Regel als Residuen nach rezidivierenden Pneumonien zu interpretieren sind. Lymphknotenmetastasen treten in bis zu $30 \%$ der Fälle auf [15].

Die Bildgebung - insbesondere die Computertomographie - hat einen festen Stellenwert in der Diagnostik von Lungentumoren. Seit ihrer klinischen Einführung 1974 durch Hounsfield konnte die Computertomographie kontinuierlich weiterentwickelt werden. 1992 wurde mit der Vorstellung des ersten Zweizeilenscanners das Verfahren der Mehrschicht-Spiral-CT etabliert, welches 1998 durch die Einführung der Vierzeilen-Geräte im Hinblick auf Akquisitions-Geschwindigkeit, Bildschärfe und Verringerung von Bewegungsartefakten weitere Verbesserung erfuhr. Heute steht mit der auf der Mehrschicht-Spiral-CT beruhenden virtuellen Bronchoskopie ein Verfahren zur Verfügung, welches eine nicht-invasive Beurteilung des Tracheobronchialsystems ermöglicht und so die Informationen des axialen Bildes in ein dreidimensionales, für den Kliniker leicht fassbares System überträgt. Obwohl bislang größere prospektive Studien zur Evaluation dieses Verfahrens fehlen, deuten erste Erfahrungsberichte an, dass 
insbesondere endobronchial gelegene Raumforderungen mit hinreichender Genauigkeit dargestellt werden können [16-18].

Unser Fall zeigt exemplarisch die bemerkenswerte Übereinstimmung des virtuellen Bildes mit dem bronchoskopischen Befund (Abb. 3a u.c). Selbst unter Berücksichtigung der Tatsache, dass die relevanten Bildinformationen zur Diagnose des Tumors bereits in den transversalen Schichten enthalten sind (Abb. 2 a u. b), ist doch festzuhalten, dass sich das virtuelle dreidimensionale Bild eignet, um den Prozess detailliert im Kontext seiner Umgebung abzubilden. Die Übereinstimmung zwischen konventionellem bronchoskopischen Bild und virtueller Endoskopie erlaubt damit die Beurteilung vermuteter Prozesse im zentralen Tracheobronchialbaum auch bei Patienten, die nicht bronchoskopiert werden können oder eine solche Untersuchung ablehnen. Weiterhin ist die Darstellung poststenotischer, konventionell bronchoskopisch nicht einsehbarer Strukturen denkbar, was bei unserem Patienten aufgrund eines bronchialen poststenotischen Kollaps allerdings nicht gelang. Schließlich kann das Verfahren der virtuellen 3D-Bronchoskopie bei der Planung interventioneller Eingriffe sowie bei der Verlaufsbeurteilung nach Interventionen angewandt werden. Zur weiteren Evaluation der Methode sind unseres Erachtens insbesondere die Studien sinnvoll und notwendig, die das endobronchiale Wachstum tumoröser pulmonaler Prozesse beurteilen. Hier sind in Zukunft wichtige Impulse für das diagnostische Prozedere bei Bronchialkarzinomen zu erwarten, etwa im Hinblick auf die Durchführung einer konventionellen Bronchoskopie mit Biopsie versus endoskopische Biopsie unter CT-Navigation.

Die auf der Existenz spezifischer Somatostatin-Rezeptoren im Tumorgewebe beruhende Octreotid-Szintigraphie mit Indium-111 oder Jod-123 hat einen wichtigen Platz in der Diagnostik der Karzinoidtumoren. Sie kann neben Angaben über die lokale Ausbreitung der Tumoren Informationen über Fernmetastasen liefern und damit das Staging ergänzen [19,20]. Die Methode weist eine relativ hohe Spezifität auf: So wurde bei $86 \%$ von Patienten mit histologisch nachgewiesenem Karzinoid eine korrekte szintigraphische Diagnose gestellt [20]. Bei der Initialdiagnostik der Karzinoide ist die Szintigraphie der Computertomographie deutlich unterlegen [21,22]. Die Fluorodesoxyglukose-Positronen-Emissions-Tomographie (FDG-PET) hat sich wegen der mangelhaften Sensitivität in der Diagnostik der Karzinoidtumoren bislang nicht durchsetzen können [23].

Bei lokalen, nicht-metastasierten endobronchialen Karzinoidtumoren ist die operative Resektion des betroffenen Lungenlappens mit begleitender Lymphadenektomie die Therapie der Wahl $[3,24,25]$. Sie verfolgt bei typischen Karzinoiden in aller Regel einen kurativen Ansatz. Bei großen, ungünstig gelegenen Tumoren kann eine Pneumektomie notwendig sein. Eine alternative Therapieform bei Stadium-1-Tumoren steht mit der thorakoskopischen Lobektomie zur Verfügung [26]. Die Prognose betroffener Patienten richtet sich in erster Linie nach der histologischen Charakteristik des Tumors [21]. Die 5-Jahres-Überlebensrate bei Patienten mit typischen Karzinoiden liegt bei ca. $75-95 \%[3,15,24,27]$.
Zusammenfassend handelt es sich beim endobronchialen Karzinoid um einen seltenen, neuroendokrinen Lungentumor. Bildmorphologisch erfolgt die Diagnose anhand der Computertomographie. Die Visualisierung kann zudem durch eine virtuelle 3D-CT-Bronchoskopie ergänzt werden, welche den Befund im Kontext des Tracheobronchialsystems plastisch darzustellen vermag. Weitere prospektive Studien sind notwendig, um die Potenziale der virtuellen Bronchoskopie - insbesondere im Hinblick auf intraluminales Wachstum von Lungentumoren - valide abschätzen zu können.

\section{Literatur}

${ }^{1}$ Godwin II JD. Carcinoid Tumors: An Analysis of 2837 Cases. Cancer 1975; 36: 560-569

2 Wang LT, Wilkins Jr EW, Bode HH. Bronchial Carcinoid Tumors in Pediatric Patients. Chest 1993; 103: 1426-1428

${ }^{3}$ Filosso PL, Rena O, Donati G et al. Bronchial Carcinoid Tumors: Surgical Management and Long-term Outcome. J Thorac Cardiovasc Surg $2002 \mathrm{Feb} ; 123(2)$ : $303-309$

${ }^{4}$ Travis WD. Pathology of Lung Cancer. Clin Chest Med 2002 Mar; 23(1): 65-81, viii. Review

${ }^{5}$ Mendonca C, Baptista C, Ramos M et al. Typical and Atypical Lung Carcinoids: Clinical and Morphological Diagnosis. Microsc Res Tech 1997 Sep $1 ; 38(5): 468-472$

${ }^{6}$ Todd TR, Cooper JD, Weissberg D et al. Bronchial Carcinoid Tumors: Twenty Years' Experience. J Thorac Cardiovasc Surg 1980; 79: 532 - 536

${ }^{7}$ Rosado de Christenson ML, Abbott GF, Kirejczyk WM et al. Thoracic Carcinoids: Radiologic-Pathologic Correlation. RadioGraphics 1999; 19: $707-736$

${ }^{8}$ Ducrocq X, Thomas P, Massard G et al. Operative Risk and Prognostic Factors of Typical Bronchial Carcinoid Tumors. Ann Thorac Surg 1998; 65: $1410-1414$

${ }^{9}$ Nessi R, Basso Ricci P, Basso Ricci S et al. Bronchial Carcinoid Tumors: Radiologic Observations in 49 Cases. J Thorac Imaging 1991; 6: 47-53

${ }^{10}$ Magid D, Siegelman SS, Eggleston JC et al. Pulmonary Carcinoid Tumors: CT Assessment. J Comput Assist Tomogr 1989; 13: 244-247

${ }^{11}$ Jeung MY, Gasser B, Gangi A et al. Bronchial Carcinoid Tumors of the Thorax: Spectrum of Radiologic Findings. Radiographics 2002; 22: $351-365$

12 Zwiebel BR, Austin JHM, Grimes MM. Bronchial Carcinoid Tumors: Assessment with CT of Location and Intratumoral Calcification in $31 \mathrm{~Pa}-$ tients. Radiology 1991; 179: 483-486

${ }^{13}$ Choplin RH, Kawamoto EH, Dyer RB et al. Atypical Carcinoid of the Lung: Radiographic Features. Am J Roentgenol 1986; 146: 665-668

${ }^{14}$ Aronchick JM, Wexler JA, Christen B et al. Computed Tomography of Bronchial Carcinoid. J Comput Assist Tomogr 1986; 10: 71 - 74

15 Gould PM, Bonner JA, Sawyer TE et al. Bronchial Carcinoid Tumors: Importance of Prognostic Factors that Influence Patterns of Recurrence and Overall Survival. Radiology 1998; 208: 181 - 185

${ }^{16}$ Finkelstein SE, Summers RM, Nguyen DM et al. Virtual Bronchoscopy for Evaluation of Malignant Tumors of the Thorax. J Thorac Cardiovasc Surg 2002 May; 123(5): 967-972

17 Rapp-Bernhardt U, Welte T, Doehring W et al. Diagnostic Potential of Virtual Bronchoscopy: Advantages in Comparison with Axial CT Slices, MPR and MIP? Eur Radiol 2000; 10(6): 981 - 988

${ }^{18}$ Seemann MD, Claussen CD. Hybrid 3D Visualization of the Chest and Virtual Endoscopy of the Tracheobronchial System: Possibilities and Limitations of Clinical Application. Lung Cancer 2001 Jun; 32(3): $237-246$

19 O’Byrne KJ, Schally AV, Thomas A et al. Somatostatin, its Receptors and Analogs, in Lung Cancer. Chemotherapy 2001; 47 Suppl 2: 78-108

${ }^{20}$ Kwekkeboom DJ, Krenning EP, Bakker WH et al. Somatostatin Analogue Scintigraphy in Carcinoid Tumours. Eur J Nucl Med 1993 Apr; 20(4): $283-292$

${ }^{21}$ Le Rest C, Bomanji JB, Costa DC et al. Functional Imaging of Malignant Paragangliomas and Carcinoid Tumours. Eur J Nucl Med 2001 Apr; 28(4): $478-482$ 
${ }^{22}$ Tabarin A, Valli N, Chanson P et al. Usefulness of Somatostatin Receptor Scintigraphy in Patients with Occult Ectopic Adrenocorticotropin Syndrome. J Clin Endocrinol Metab 1999 Apr; 84(4): 1193-1202

${ }^{23}$ Marom EM, Sarvis S, Herndon 2nd JE et al. T1 Lung Cancers: Sensitivity of Diagnosis with Fluorodeoxyglucose PET. Radiology 2002 May; 223(2): $453-459$

${ }^{24}$ Carretta A, Ceresoli GL, Arrigoni G et al. Diagnostic and Therapeutic Management of Neuroendocrine Lung Tumors: A Clinical Study of 44 Cases. Lung Cancer 2000 Sep; 29(3): 217-225
${ }^{25}$ Terzi A, Lonardoni A, Falezza G et al. Sleeve Lobectomy for Non-Small Cell Lung Cancer and Carcinoids: Results in 160 Cases. Eur J Cardiothorac Surg 2002 May; 21(5): 888-893

${ }^{26}$ Daniels LJ, Balderson SS, Onaitis MW et al. Thoracoscopic Lobectomy: A Safe and Effective Strategy for Patients with Stage I Lung Cancer. Ann Thorac Surg 2002 Sep; 74(3): 860 - 864

${ }^{27}$ Perkins P, Kemp BL, Putnam Jr JB et al. Pretreatment Characteristics of Carcinoid Tumors of the Lung which Predict Aggressive Behavior. Am J Clin Oncol 1997 Jun; 20(3): 285-288

\section{Buchbesprechung}

\section{Arbeitsmedizin. Handbuch für Theorie und Praxis}

G. Triebig, M. Kentner, R. Schiele (Hrsg)

Stuttgart: Gentner Verlag, 2003. 1174 S. €128,-

Zahlreiche zum Teil sehr namhafte Fachleute der Arbeitsmedizin aus universitären Instituten, Praktiker aus arbeitsmedizinischen Organisationen, Industriebetrieben, Aufsichtsbehörden und Berufsgenossenschaften, unterstützt von Juristen haben sich der Herausforderung gestellt, ein Lehrbuch der Arbeitsmedizin für Studierende und angehende Arbeitsmediziner zu schreiben, das auch gleichzeitig für andere medizinische und nicht-medizinische Bereiche ein Nachschlagewerk darstellen soll.

Nach kurzer Einführung in das Gebiet werden zunächst die umfangreichen gesetzlichen Grundlagen erörtert, wobei das Problem der sich ständig ändernden und weiterentwickelnden Gesetze und Vorschriften mit Übergangsbestimmungen gelöst wird durch Verweis auf Internetadressen, bei denen die aktuellen Informationen abzurufen sind.

Die umfassende Abhandlung der Berufkrankheiten, oft erläutert durch Beispiele, weist den wichtigen Zusammenhang mit der Sozialmedizin auf, wozu auch die Kapitel der Rehabilitation und Prävention beitragen. Arbeitsphysiologie, Ergonomie, Arbeitspsychologie und besondere Arbeitsplatzbelastungen werden leicht lesbar und verständlich auch für Nicht-Arbeitsmediziner besprochen.
Die spezifische arbeitsmedizinische Methodik zur Beurteilung der Einsatzfähigkeit von Arbeitnehmern im Hinblick auf die Beanspruchung der einzelnen Organsysteme wird ausreichend erläutert und von klinisch diagnostischen Methoden abgegrenzt, ergänzt durch die Methoden des Biomonitorings und der Gefährdungsanalyse bei genetischer Disposition.

Die Geschichte der Arbeitsmedizin findet sich ungewöhnlicherweise am Ende ebenso wie die Umweltmedizin und das Autorenverzeichnis.

Das große Schriftbild, die klare Gliederung und die kurzen $\mathrm{Zu}$ sammenfassungen am Ende eines jeden Kapitels machen das Lesen leicht, das farbige Unterlegen von Schlüsselsätzen erleichtert das Erlernen und Behalten des Wesentlichen, die Angabe der weiterführenden Literatur macht eine Vertiefung besonderer Fragen einfach.

Kleinere Fehler im Kapitel Silikotuberkulose fallen einem Pneumologen auf (beispielsweise bei Diagnostik und Therapie dieser Berufskrankheit), sollten auch bei einer absehbaren Neuauflage korrigert werden, schmälern aber nicht den insgesamt sehr positiven Eindruck eines Buches über ein lange etwas stiefmütterlich behandeltes Fachgebiet.

H. Steveling. Essen 